\title{
3D structural characterisation, deformation measurements and assessment of low-density wood fibreboard under compression: The use of X-ray microtomography
}

\author{
E. Badel ${ }^{\mathrm{a}, *}$, C. Delisee $^{\mathrm{b}}, \mathrm{J}_{\text {. Lux }}{ }^{\mathrm{c}}$ \\ ${ }^{a}$ INRA, LERMAB, 14 rue Girardet, 54000 Nancy, France \\ ${ }^{\mathrm{b}}$ US2 B, Domaine de l'Hermitage, 69 Route d'Arcachon, 33610 Cestas Gazinet, France \\ ${ }^{c}$ LEPTAB, Avenue M. Crepeau 17042 La Rochelle, France
}

Received 13 September 2007; received in revised form 30 January 2008; accepted 10 February 2008

Available online 15 February 2008

\begin{abstract}
A low-density wood fibreboard has been compressed along its transversal direction. The experiment was carried out in ESRF synchrotron (ID19) and X-ray microtomographic images were recorded for each state. Stipulating that the fibreboard is a discontinuous material essentially made of air, that the compression simply re-organises the spatial distribution of the fibres and does not involve their intrinsic mechanical properties, we are able to deduce the material points density variations along the thickness of the panel. Good agreement is achieved between the macroscopic deformation of the sample and the microscopic compression rate evaluation. Then, the modifications of structural parameters are investigated by 3D image analysis. The relationship between the local structure and the behaviour of the wood fibreboard are deduced. Finally, a modelling approach allows the local densification to be predicted and confirms the initial hypotheses about the local behaviour of the material. In particular, polyester bonds are not involved.
\end{abstract}

(c) 2008 Elsevier Ltd. All rights reserved.

Keywords: A. Fibre; B. Compression; C. Deformation; D. Non-destructive testing; D. X-ray tomography

\section{Introduction}

According to new normative laws, the designers pay more and more attention to the acoustic noise reduction and the thermal insulation of the buildings. For that purpose, the use of low-density material is clearly increasing. Because of their good thermal and acoustic performances and their very low cost, the insulation panels are still primarily made of glass wool (or rock wool). Their thermal, acoustic and mechanical properties have been widely studied to improve the manufacturing process $[1,2]$. The growing need for environmental friendly materials has stimulated an increasing interest in the natural fibreboards, and especially wood fibreboards. These are made of single

\footnotetext{
${ }^{*}$ Corresponding author. Tel.: +33 3833968 86; fax: +33 383396847.

E-mail addresses: badel@nancy.inra.fr (E. Badel), delisee@us2b.pierroton.inra.fr (C. Delisee), jerome.lux@univ-lr.fr (J. Lux).
}

fibres and bundles that are laid on a conveyor belt. Additional glue is usually used to ensure the cohesion when the panel enter in the hot press. Their technical properties however remain largely unknown. In the last few years, numerous studies have been initiated to characterize the behaviour and to understand the relationships between the nature, the size, the spatial organisation of the fibres and the properties in order to improve the manufacturing process. Most of these are dedicated to thermal properties [3-5]. Since these materials are not used as structural elements, there is little literature about the mechanical deformation of the low-density fibreboard [6] under mechanical stress, whereas the properties of singles fibres (rupture, elastic and visco-elastic properties) are widely studied for other wood based composite materials, paper or massive wood assessment purposes [7-11]. However, the deformation under stress behaviour is involved during the storage in the manufactures or at the retailers and during the 
handling in the building sites. In this paper we investigate the compression of a low-density wood fibreboard perpendicularly to its main faces and the relationship between its behaviour and the local structure. This is observed in situ thanks to high-resolution X-ray microtomography that allows the organisation of the fibres to be followed in $3 \mathrm{D}$ during the compression test without external perturbation.

\section{Material and method}

\subsection{Material}

The material sample was taken from very low-density wood fibreboard used for thermal insulation of the building. It is composed of maritime pine fibres and bi-components thermo-fused polyester fibres to ensure cohesion. Wood fibres are composed of isolated fibres (average diameter and length around $40 \mu \mathrm{m}$ and $1 \mathrm{~mm}$ ) and bundles (aggregation of several fibres, with diameter and length up to $500 \mu \mathrm{m}$ and $10 \mathrm{~mm}$ ), showing strong variability of sizes. The diameter and length of polyester fibres are, respectively, $20 \mu \mathrm{m}$ and $55 \mathrm{~mm}$. A non-woven textile process, adapted to wood fibre based materials manufacture, produces a thick board with a 3D architecture. Before consolidation, the fibres are distributed and randomly oriented. Then the mat is rolled out at the required thickness and is consolidated in a kiln. This induces a main orientation along planes parallel to the faces [5]. The cohesion of this discontinuous matter is provided by fibre-to-fibre connections thanks to the fusion of plastic fibres (Fig. 1). The average macroscopic gravimetric density of the material is about $45 \mathrm{~kg} \mathrm{~m}^{-3}$. A small cylinder was carefully extracted from the board for the mechanical test. The diameter and height were, respectively, around $10 \mathrm{~mm}$ and $12 \mathrm{~mm}$.

\subsection{Compression tests}

The compression experiments were carried out in the Xray microtomographic device installed at the ID 19 beam

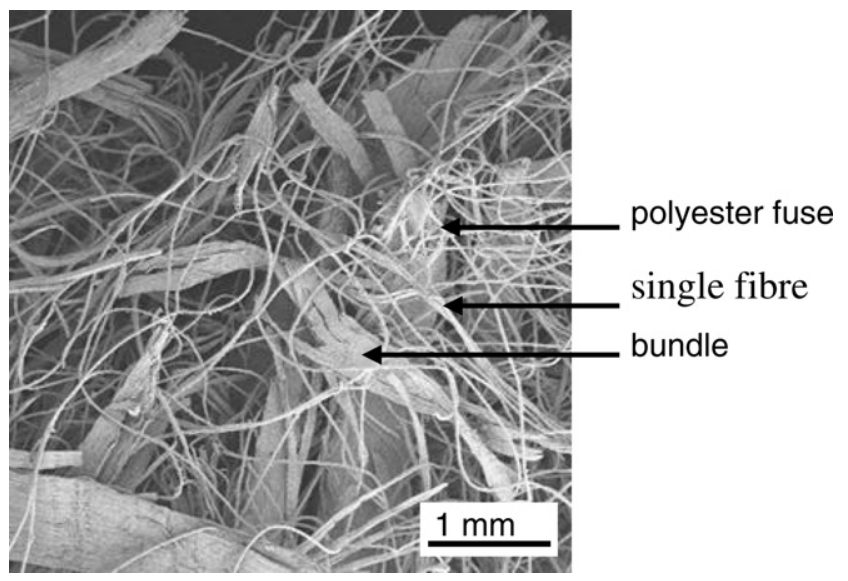

Fig. 1. SEM image of wood fibreboard. The wood bundles and fibres are connected by the thin polyester bonds. line of the European Synchrotron Radiation Facility (ESRF, France). A total of $2048^{3}$ voxels volumes $\left(\approx 10.05^{3}\right.$ $\mathrm{mm})$ with a $4.91 \mu \mathrm{m}$ spatial resolution were recorded. The grey level value of each voxel represents the average attenuation properties in this voxel.

Mechanical tests were conducted in a specially designed tensile/compression testing machine lent by the GEMPPM laboratory [12]. It is composed of two horizontal plates: one is fixed while the second is mobile and vertically translated with a motor. A PMMA tube transmits the vertical load (Fig. 2) perpendicularly to the surface of the panel. The sample is constrained in the tube (internal diameter equal to $10 \mathrm{~mm}$ ) guaranteeing macroscopic 1D displacement. A pre-load was performed to ensure good contact between the sample and the plates. Then, two tomographic scans were recorded at two states of compression called "initial" and "compressed". The experimental macroscopic deformation was equal to $-32.8 \%$.

\subsection{Crop of the $3 D$ volumes}

Sub-volumes were extracted from the raw data in the core of the sample, both to reduce the size of the file for its analysis and to avoid surface modifications induced by the material cutting. The final sizes of the sub-volumes were $1024^{2} \times 1360$ pixels for the initial and $1024^{2} \times 973$ pixels $^{3}$ for the compressed states. They are larger than the size of a REV (representative volume element) as previously estimated for this material by Lux [5].

\subsection{Structural measurements}

The visualisation and the basic image treatments were performed using ImageJ [13]. The structural measurements by $3 \mathrm{D}$ image analysis $[14,15]$ were performed using Aphelion $^{\mathrm{m}}$. Morphological operations were used to characterize the two states of compression in different ways. First, the 3D images were thresholded in order to separate the solid phase and the air. Then, basic operations were applied to a set (of fibres or of pores). They are dilation and erosion by structuring elements that are reference sets of given geometry (segments, spheres, cubes, octahedrons, etc...). Usual composed operations are opening and closing which are, respectively, an erosion followed by a dilation and a dilation followed by an erosion.

\subsubsection{Porosities}

The total porosity, i.e., the volume fraction of air in the entire sample, was measured. It includes the air fraction outside the fibres and their internal porosity. The fibre porosity is related to the volume fraction of internal porosity (lumens included) in the wood particles. It is expressed in percentage of the volume of filled fibres. The fibres were filled (Fig. 3) using morphological operations [5]. Finally, it gives the porosity referred as external porosity, i.e., the volume fraction of air lying outside of the filled fibres. 

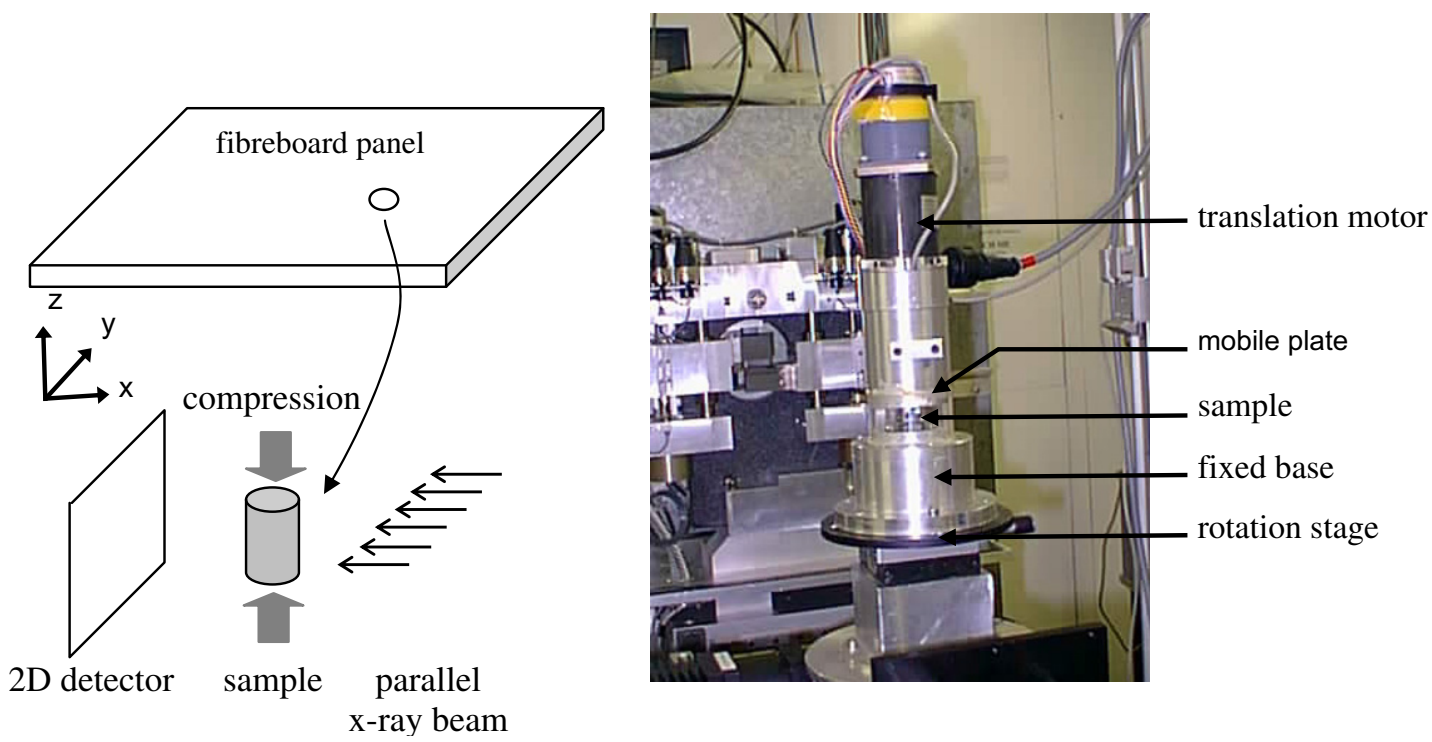

Fig. 2. The compression testing machine installed on the ID 19 X-ray beamline (ESRF-France). $x$ and $y$ directions refer to the main directions of the panel and are called "transversal" directions. $z$ refers to the direction of the compression and is called "vertical" direction.
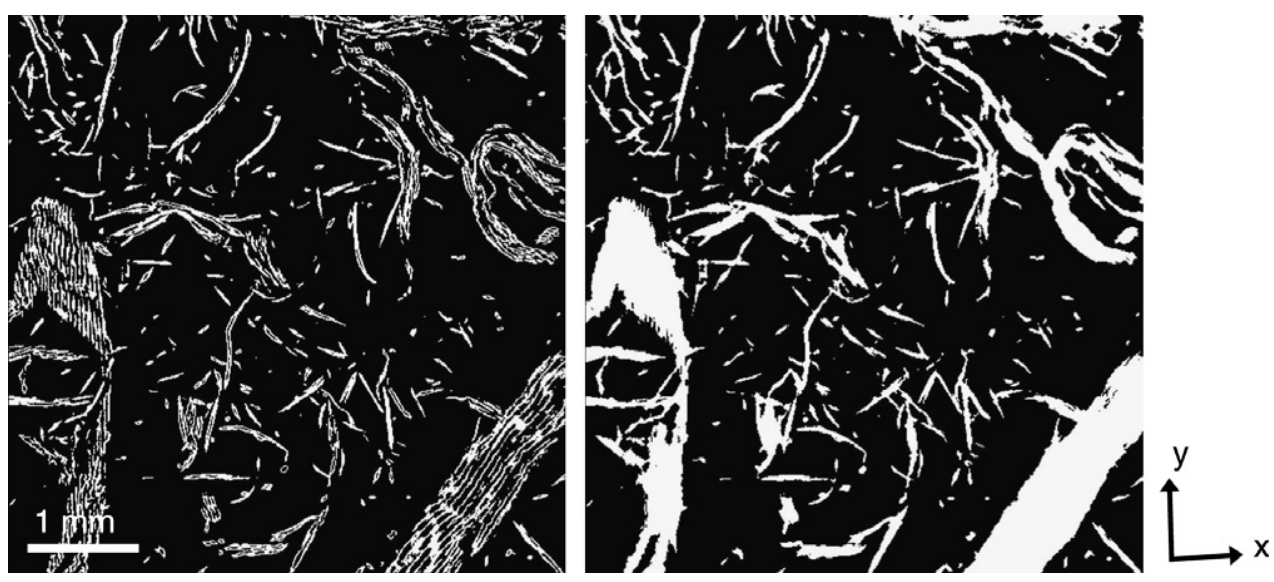

Fig. 3. The filling of the fibres. 3D morphological operations are used to fill the internal porosity (lumens of the cells).

\subsubsection{Granulometry}

The size distribution of a set of fibres (or pores) was assessed by performing successive openings [15] by a structuring element of growing size. It consists of measuring the volume fraction of the set that is smaller than the chosen structuring element. The cumulated size distribution $G(X, \lambda)$ of a set $X$ is defined by

$G(X, \lambda)=1-\frac{V\left(\gamma_{B_{\lambda}}(X)\right)}{V(X)}$

with $B_{\lambda}$ the structuring element of size $\lambda, V(X)$ the measurement of the volume of the set and $\gamma_{B_{\lambda}}$ the opening by $B_{\lambda}$.

Considering the high connectivity of the pores and their varied shapes, their granulometry gives information about the inter-fibres distance. Moreover, as the fibres are rather oriented in planes parallel to the faces, it gives information about the inter-fibres distance along the direction of compression.
Granulometric curves are computed in a similar way for the set of the filled fibres. It allows the measurement of their diameters.

\subsubsection{Auto-covariance}

The auto-covariance function $C(h, \alpha)$ of a set $X$ is related to the probability of a voxel (position $x$ ) to be in the same phase as another voxel (position $x+h_{\alpha}$ at a distance $h$ and orientation $\alpha$ from $x$. Considering the set $X_{\alpha}$ translated of $X$ by $h_{\alpha}$, the auto-covariance function may be estimated as a measure of the number of voxels in $X \cap X_{h_{\alpha}}$ over the sum of all over possibilities as given by

$C(h, \alpha)=P\left\{x \in X ; x+h_{\alpha} \in X\right\}-X_{V}^{2}=\frac{V\left(X \cap X_{h_{\alpha}}\right)}{V\left(Z \cap Z_{h_{\alpha}}\right)}-X_{V}^{2}$

with $V(X)$ the volume of $X$ and $X_{V}$ the volume fraction of $X$. 
It allows the detection of a possible anisotropy of the structure [14]. The correlation between two voxels at a distance $h$ actually depends on the orientation $\alpha$. Next, we call the "covariogram" the representation of $C(h, \alpha)$ along one fixed direction.

Auto-covariance functions are computed on the set of the fibres. In order to clearly see the relevant information, only a few covariograms are plotted, but the analysis is based on the total auto-covariance function.

\subsection{Following of the local material point density}

The following of the compression rate is based on the position of the material points of the wood particles. This involves usually more or less complex techniques using image correlation methods [2,16-21].

We decided to use the specificities of the fibreboard material here. First, observations show very few inter-connexions between the fibres. Apart from the very thin polyester bonds, the material appears as discontinuous matter. We expect that the wood fibres and bundles can move by solid displacement involving no internal stress. This is the second key point: this very low-density material is mainly composed of air volumes that give much space for the fibres and bundles to move, so we stipulate that the fibres

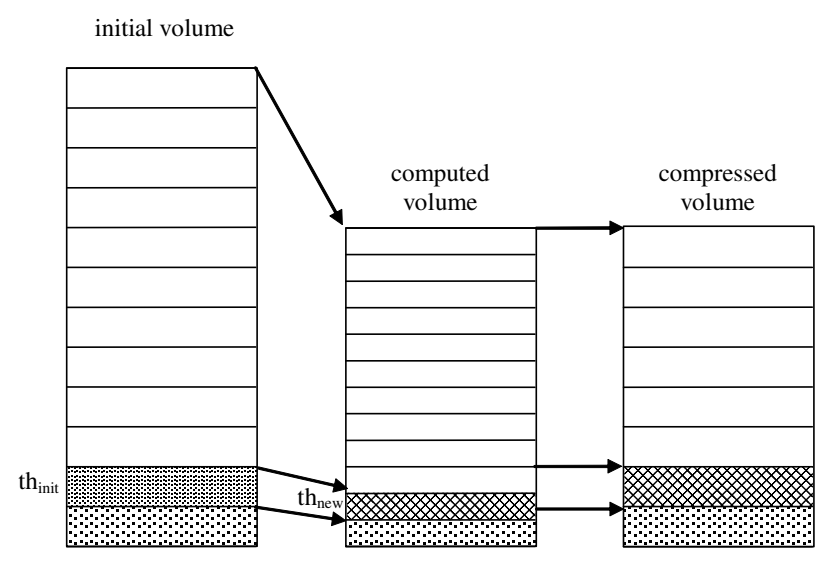

Fig. 4. 1D local re-distribution of the material points. Computation of the individual slice compression rate. and bundles are not individually compressed. The macroscopic deformation of the board generates only a new organisation of the fibres in the structure. The density we are attempting to follow becomes a material point density of wood particles. The number of material points in a finite volume becomes the natural marker of this density. The densification process is as follows.

After thresholding, the volume is split into transversal slices, one voxel thick, which are perpendicular to the compression direction. The number of material points is integrated on each slice and is plotted along the vertical $(z)$ direction for the initial and compressed states. These 1D profiles are used to evaluate the "local" compression rate as follows: since the number of material points is well known in the first slice in the initial state, the corresponding volume is computed in the compressed state in order to ensure the material points conservation. This determines a new thickness of the $i$ th slice and the compression strain is evaluated according to the following equation (Fig. 4):

$\varepsilon(i)=\frac{\operatorname{th}_{\text {new }}(i)-\operatorname{th}_{\text {init }}(i)}{\operatorname{th}_{\text {init }}(i)}$

with thew $h_{\text {new }}$ the new computed thickness and th $\mathrm{h}_{\text {init }}$ the initial thickness of the slice. Starting from the new top positions, the process is iterated along the vertical direction up to the top of the sample. The starting point is determined using the fixed solid support as a reference, which is not affected by the low stress during the compression of the sample.

\section{Results and discussion}

\subsection{Visualisation and morphology}

Because it provides information about the local structure, visualisation is an important step. We visualize the sample using 3D rendering or by extracting $2 \mathrm{D}$ slices. This very low-density material is very fragile and is therefore very difficult to examine: the cutting breaks the entangled structure and the surface is always damaged, so, X-ray tomography is a very efficient non-destructive tool to observe the material in situ.

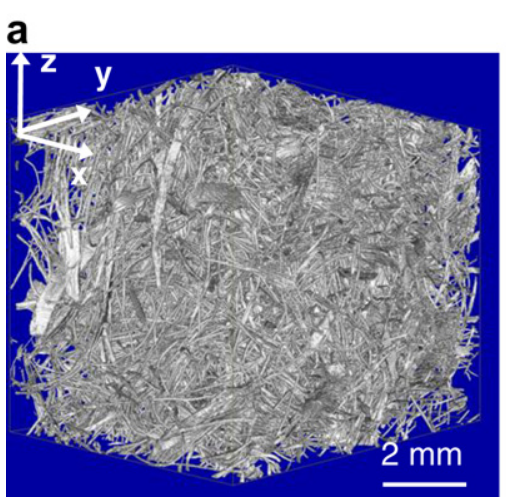

b

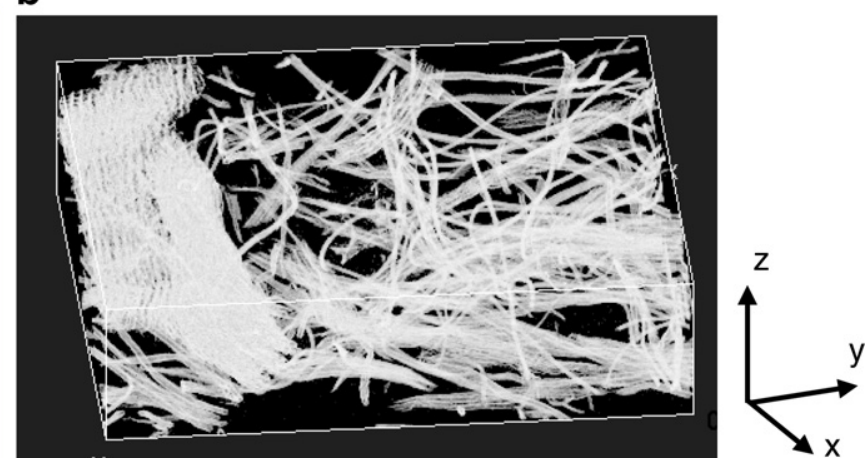

Fig. 5. 3D visualisations of the wood fibreboard. (a) Entire volume (non-compressed) and zoomed view (b). The $z$ direction refers to the compression direction (perpendicularly to the panel). $x$ and $y$ directions refer to the transversal directions. 
Fig. 5a and $\mathrm{b}$ depict two examples of visualisation by $3 \mathrm{D}$ rendering. Fig. 5a depicts the complete volume, while Fig. 5b exhibits the complex 3D organisation of the fibres in the material and reveals the microscopic heterogeneity. Most of the space is filled by the entangled isolated single fibres but the mass is concentrated in bundles. This leads to a great heterogeneity of the density distribution in the volume.

The $3 \mathrm{D}$ observations bring a qualitative overview of the microscopic and mesoscopic structure, especially the shape and size of the particles. $2 \mathrm{D}$ views allow other parameters to be evaluated. Fig. 6a is a vertical slice. It highlights the very low part of the space that is occupied by the fibres and their intrinsic porosity. This cannot be observed on the (xy) transversal planes (Fig. 6b) and suggests that the main orientation of the fibres is along the transversal planes. So, the length of the single fibres can be observed on these transversal views whereas the vertical slices show their section. This structural specificity, due to the manufacturing process, is in accordance with previous works about the fibre's orientation $[5,22]$.

Considering the values of the porosities measured for the two states (Table 1), the total porosity reduces from $92.4 \%$ to $88.6 \%$ and the external porosity reduces from $87.8 \%$ to $81.8 \%$; whereas internal fibres porosity remains constant. The morphology of single fibres and bundles seems not to be affected by the compression process.

In the same way, granulometric curves, computed, respectively, for the pores and for the fibres (Fig. 7), show that the size distribution of the fibres is not modified by the compression process, contrary to the size distribution of the pores.

Considering the orientations of the fibres, Fig. 8a shows covariograms computed for five directions for the initial state: one along the compression axis and four along directions in the transversal plane. First, the fibre distribution is isotropic in the transversal plane, since all the corresponding curves are very close. Secondly, the curve corresponding to the direction of compression is below all the other
Table 1

Porosities for the two states as measured by $3 \mathrm{D}$ image analysis

\begin{tabular}{llll}
\hline $\begin{array}{l}\text { State of } \\
\text { compression }\end{array}$ & $\begin{array}{l}\text { Total } \\
\text { porosity (\%) }\end{array}$ & $\begin{array}{l}\text { Internal fibres } \\
\text { porosity (\%) }\end{array}$ & $\begin{array}{l}\text { External } \\
\text { porosity (\%) }\end{array}$ \\
\hline Initial state (comp 1) & 92.4 & 37.1 & 87.8 \\
Compressed state (comp 2) & 88.6 & 37.4 & 81.8 \\
\hline
\end{tabular}

The total and external porosities references to the entire sample. The internal fibre porosity references to the volume of the filled fibre.

curves. These two observations underline that the fibres are mainly oriented along transversal planes, but are randomly oriented in them. This is in agreement with the manufacturing process. The same conclusions apply for the compressed state.

Let us now compare the two mechanical states. Fig. $8 \mathrm{~b}$ shows, for both states, a comparison between the autocovariance curves normalized by the variance, for only three directions (two in the transversal plane and one along the compression axis). As a first remark, the covariograms corresponding to the same direction are very close and the relative importance of each one is preserved. There is consequently no major modification of the underlying structure between the two compression rates. Since the relative difference between the curves is preserved, the anisotropy between the vertical and horizontal directions is not affected by the compression. However, the normalized covariograms corresponding to the compressed state are systematically below those corresponding to the initial state. It is somewhat intuitive. The normalized auto-covariance can be expressed as follows:

$$
\frac{C(h, \alpha)}{C(0)}=\frac{P\left\{x+h_{\alpha} \in X / x \in X\right\}-X_{V}}{1-X_{V}}
$$

Thus the auto-covariance is a measure of the difference between the raw conditional probability $P\left\{x+h_{\alpha} \in X / x \in X\right\}$ and the volume fraction $X_{V}$ (which is equal to $P\{x \in X\}$ ). We observe here that the raw probability to find two points in the same phase is slightly higher in the compressed state, due to the higher density, but not enough so that it a

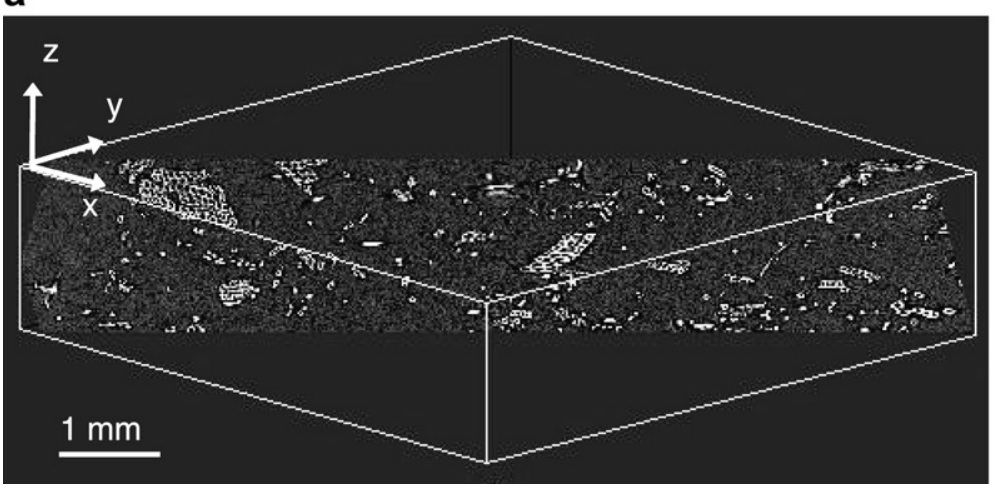

b

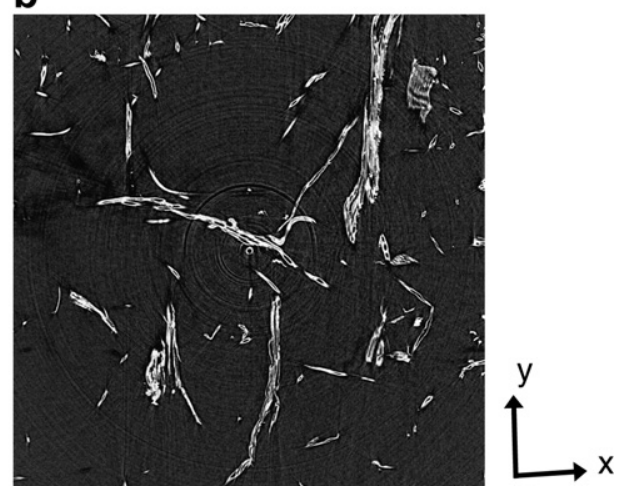

Fig. 6. 2D unit slices (1 voxel thick) extracted from the fibreboard. (a) Vertical slice and (b) transversal slice ( $x y$ ). The wood fibres occupy very little space. According to their preferential orientation, the lumens of the cells are clearly observable on the vertical slices while the length of the fibres appears in transversal slices. 
a

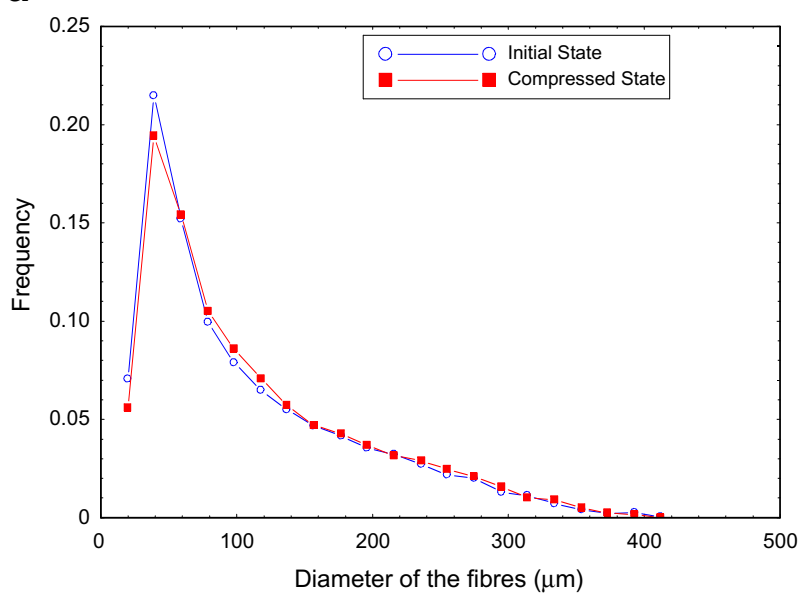

b

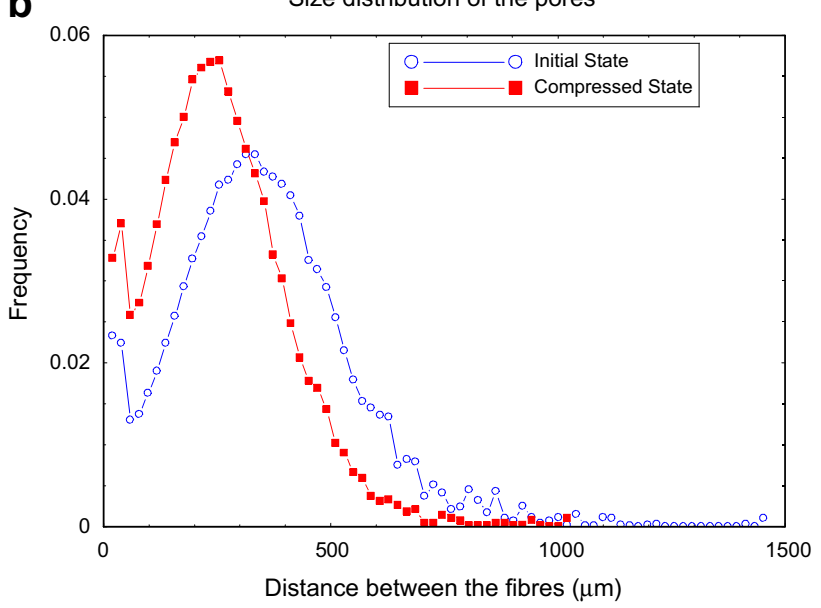

Fig. 7. Granulometric measurements. The dimensions of the fibres are not modified by the compression (a), while distance between the fibres decreases (b).

a

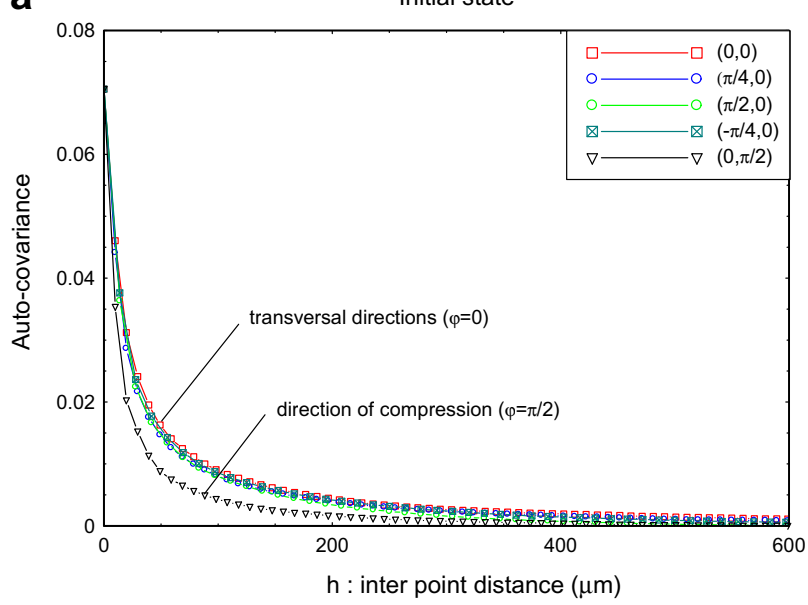

b

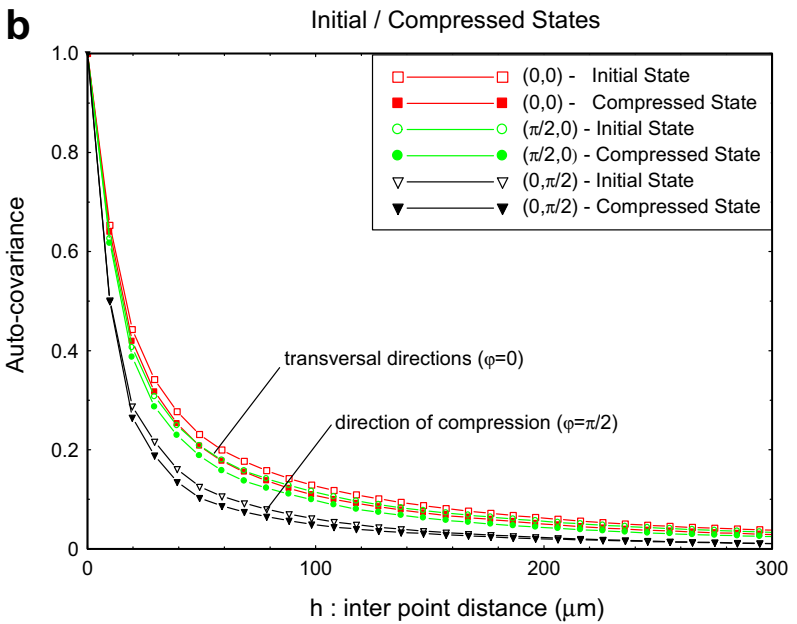

Fig. 8. Covariograms computed for the set of the fibres. (a) Covariograms in different directions of the fibreboard (initial state only). Directions are expressed in spherical notation $(\theta, \varphi) . \varphi=0$ references to the transversal plane and $\varphi=\pi / 2$ references to the compression axis. (b) Comparison between initial and compressed states (the covariograms have been normalized for comparison).

overcomes the increase of $X_{V}$. In other words, the correlation between two points decreases. If it is observed that the differences between the two series of covariograms concerns only short range correlation (less than $200 \mu \mathrm{m}$ on transverse plane and less than $100 \mu \mathrm{m}$ for vertical direction), then this phenomenon is an indication that there is no sensible modification of individual fibres, be it either compression, agglomeration or orientation at these compression level. Sensible effects of a different character may appear at higher compression rates.

\subsection{Material point density}

Fig. 9 depicts two corresponding transversal slices very close to the compression plate; one at the initial state and the second at the compressed state. Many particular points correspond on the both images. However, the density is higher on Fig. 9b. Two different factors contribute to this increase. On the one hand, the number of fibres mounts significantly in the slice; new fibres or part of them, coming from the neighbouring top slices move to the bottom. On the other hand, the previous visible bundles appear larger. This should be linked to the previous analysis of the comparison of the covariogram applied to the $(0, \pi / 2)$ orientation. So, a larger proportion of the bundle can be observed in the slice. The same comment is available for the single fibres that appear longer.

The profiles of density distribution along the vertical direction bring some information (Fig. 10). The heights of the sample can be evaluated; and the displacement of the mobile plate as well. The spatial resolution of the measured distances is about one voxel, i.e., about $5 \mu \mathrm{m}$. The macroscopic deformation of the entire sample $\varepsilon_{12}$ is then evaluated to about $32.6 \% \pm 0.06$. The profiles show clearly 
a

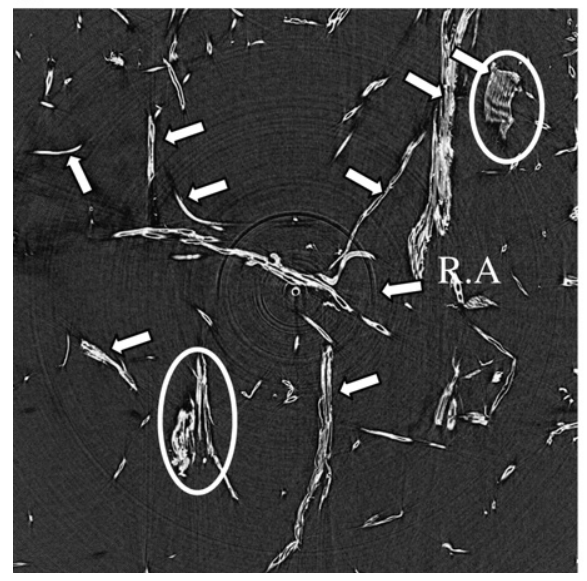

b

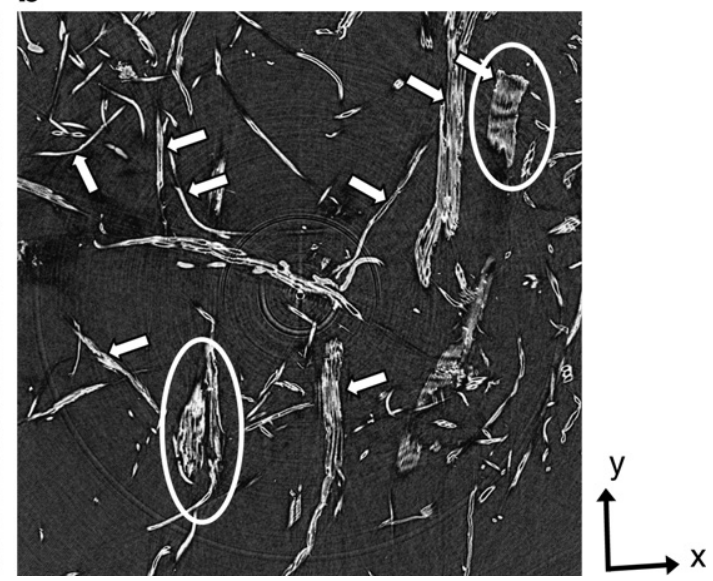

Fig. 9. Visualisation of two transversal slices $(x y)$. Grey level images are extracted from the "initial" (a) and "compressed" (b) volumes. The number of single fibres increases. The sizes of the bundles grow as well (surrounded areas).
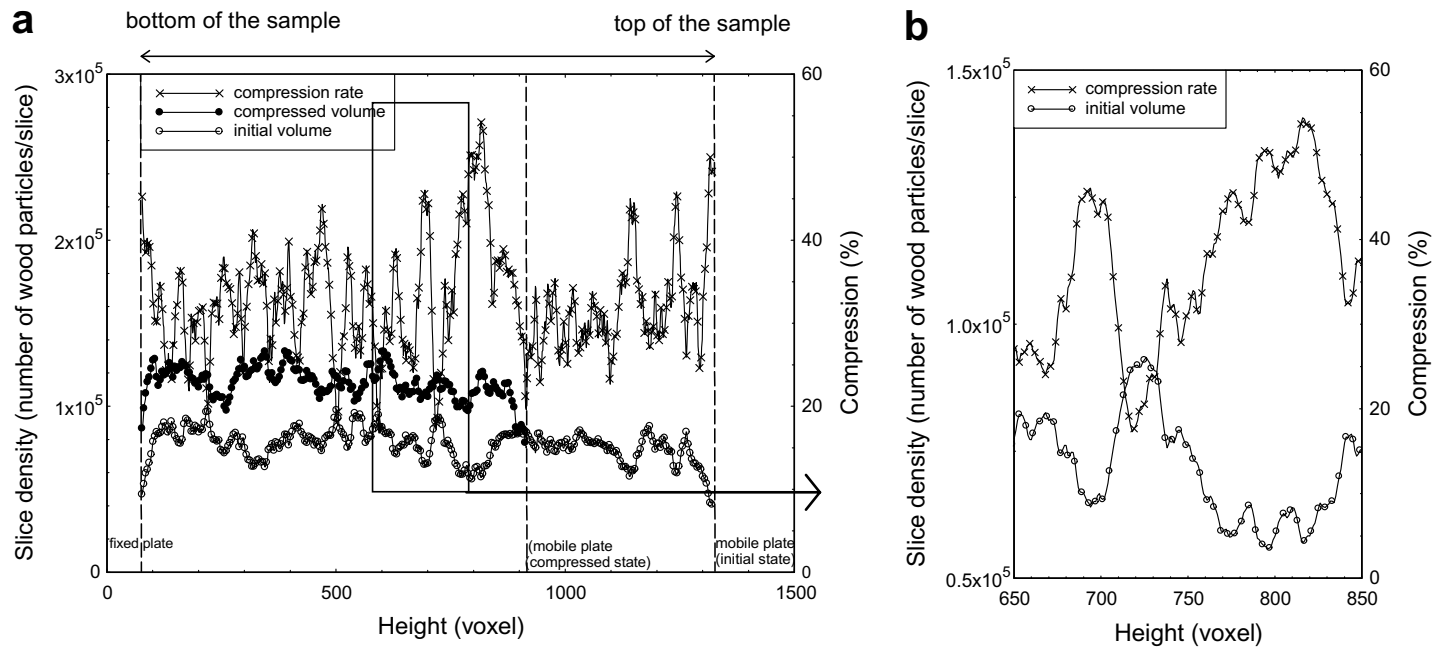

Fig. 10. The material point densification along the compression direction (a). The positions of the bottom and top supports aid the determination of the starting point and endpoint. The compressed profile is slightly more even. The compression rate varies locally as the inverse of the initial density (b).

the increase of the slices' density. The initial heterogeneity of the material points density is highlighted too but no significant gradient is observable. Nevertheless, the material appears as a very irregular multi-layer composite made of soft and denser layers. This results mainly from the presence or absence of bundles.

Using the incremental process see Section 2.5 and Eq. (3), the local densification is evaluated along the thickness (Fig. 10). The macroscopic compression of the entire sample is computed equal to $32.77 \%$. This has to be compared to the previous manual macroscopic evaluation $(32.6 \%)$. This proves two points. On the one hand, after 1246 iterations, the process computes itself almost the same top slice, as we did manually ( 1 slice difference). This is validated by visual observation of the last slices. These values are also in total agreement with the experimental data we measured during the compression experiment $(32.8 \%)$. On the other hand, this corroborates the initial hypothesis of the material points conservation:
- It confirms the unidirectional displacement of the matter. There is no lateral matter transfer from the studied sub-volume to other parts of the sample.

- It confirms the non-compression of the solid fraction; neither for the singles fibres nor for the bundles.

Fig. 10b shows that a local relationship between the initial material points density and the compression rate can be suspected. The fluctuation of the local densification varies as a negative function of the initial density. This influence of the initial state is highlighted as the local densification is plotted versus the initial density (Fig. 11). This correlation confirms that the material deforms as a multi-layer material made of series of low and higher rigidity areas. Obviously, the deformation becomes higher in the lower density parts during the compression. In case of our material points tracking, it indicates that the particles move preferentially into the low-density area to fill them. 


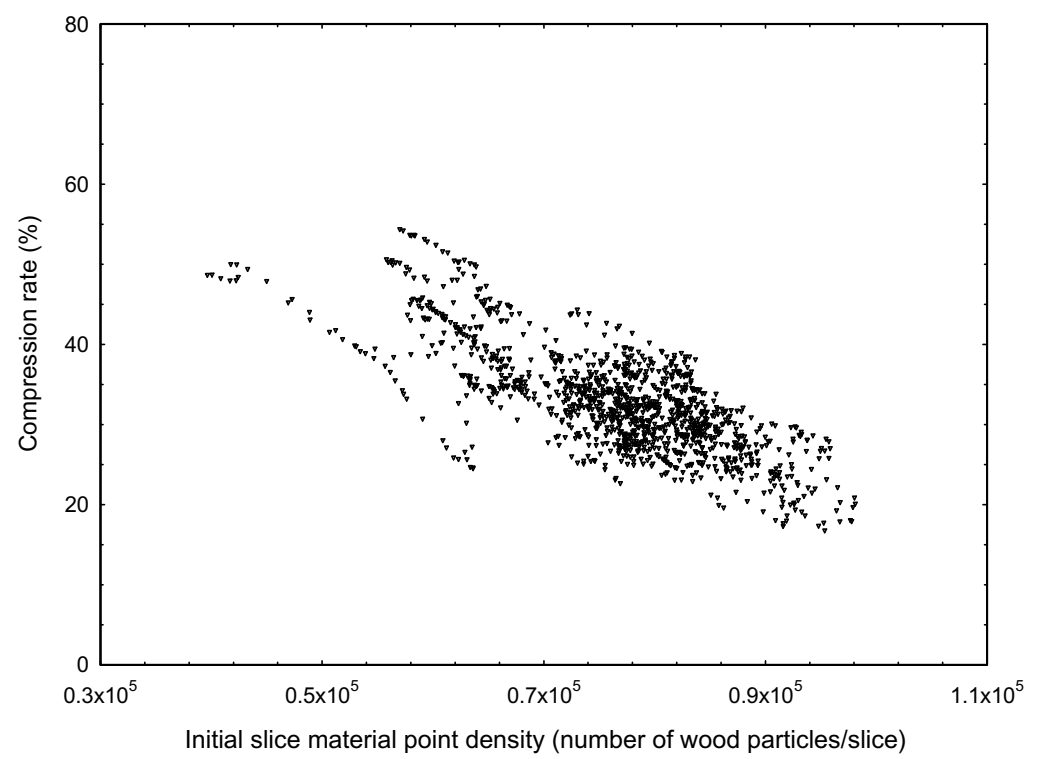

Fig. 11. Compression level versus the initial material point density. The lower density slices play a greater role in the displacement fields.

\subsection{Modelling the local deformation}

All the previous observations lead to the hypothesis that the fibreboard behaves as a discontinuous material. However it has been verified by SEM observation that the fibres are well connected by the polyester fibres (Fig. 1). In fact, we can easily presume that, in case of compression, the thin bonds, which have a very ductile behaviour, act as wires. They bring neither bending, nor compression stress during the test and the deformation occurs as if they were not present. If the deformation really occurs only in the air fraction, we could write

$$
\varepsilon_{12}=\frac{P_{2}-P_{1}}{1-P_{2}}
$$

Since, the porosities $P_{1}$ and $P_{2}$ have been measured by image analysis (Table 1), this equation leads to a theoretical compression rate equal to $-32.8 \%$. It is totally in accordance with all the previous evaluations. Moreover, if the wood fibres are not individually compressed, then their internal porosity should not change. Again, this is verified by the image analysis measurements (Table 1).

Now, we propose a simple modelling of the local deformation of the fibreboard that requires only the knowledge

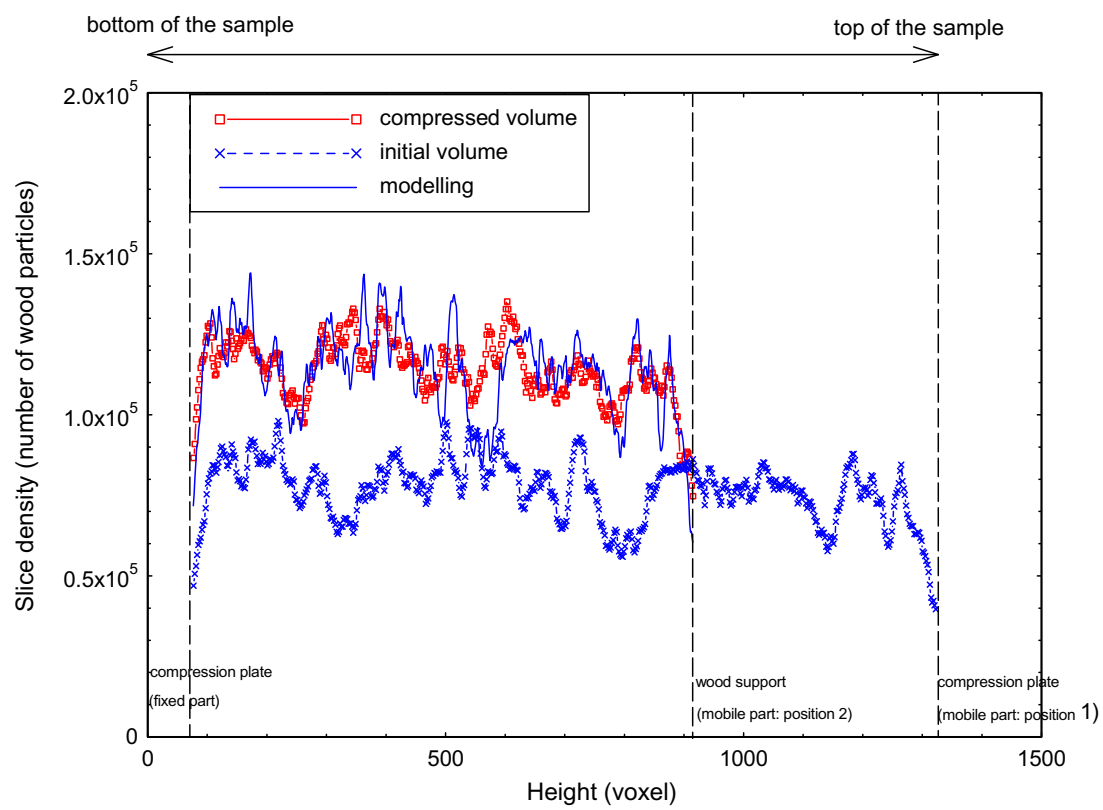

Fig. 12. Modelling the local deformation of the fibreboard. The initial profile of the spatial distribution of the wood particles is the input of the model. The computation is compared to the experimental profile that is measured on the microtomographic data. 
of the macroscopic deformation $\varepsilon_{12}$ and the distribution of the local porosity $p(i)$ (or wood particles volume) as input parameters. At the macroscopic level (entire sample), the relationship between the deformation of the air fraction and the sample's deformation can be written

$\varepsilon^{\text {air }}=\frac{\varepsilon_{12}}{p}$

At the local level, the relation can be written for each slice $i$

$\Delta \operatorname{th}^{\text {air }}(i)=\varepsilon^{\text {air }} \operatorname{th}^{\text {air }}(i)=\varepsilon^{\text {air }} p(i)$

Finally, the new thickness of each slice can be computed as follows:

$\operatorname{th}_{\text {new }}(i)=\operatorname{th}_{\text {initial }}(i)+\Delta \operatorname{th}^{\text {air }}(i) \quad$ with $\operatorname{th}_{\text {initial }}(i)=1 \forall i$

The new distribution of the wood particles is computed for each slice along the thickness of the entire sample and the number of particles is redistributed into slices having a unit thickness in order to compare with the microtomographic data. We computed the Eq. (8) with $\varepsilon_{12}=-0.327$. Fig. 12 shows the distribution computed by the modelling. The comparison with the experimental data is quite good.

- The global shape of the curve is recovered, even if a little zone (from $z=500$ to $z=600$ ) is not very well described in the middle of the sample. This could be due to the presence of a big bundle that does not match the hypothesis.

- The total size of the sample in the new compressed configuration is recovered.

These agreements confirm all previous hypotheses and allow a good prediction of the local deformation to be performed. Nevertheless, 3D modelling, that takes the position and the size of each particle into account, is under progress in order to separate the case of isolate fibres and of the bundles.

\section{Conclusion}

X-ray microtomographic images have been performed in order to characterize the internal structure of very fragile low-density wood fibreboard. The quality of the images allowed the following of the material points distribution during a $1 \mathrm{D}$ compression test. Assuming that the material is mainly composed of air and its microstructure is almost discontinuous, a specific 1D treatment has been carried out to compute the local deformation. The good agreement between the experimental setup, the visual observations on the images and the results of the computation confirm the relevance of the initial assumptions; especially the vertical displacement of the particles and the material points conservation. It suggests that the microstructure of this very low-density material re-organises the distribution of the single fibres and of the bundles in the large free volumes, without individual compression of the fibres (or bundles). This is in accor- dance with the characterisation by image analysis performed for the two compression states, in particular the measurements of the internal porosities. In those conditions the external porosity becomes essential. Assuming that the deformation occurs only in the air fraction and that the fibreboard reacts as a discontinuous material, a simple mechanical model has been proposed. The good agreement between the computed local distribution of the wood particles in the compressed state and the microtomographic observation confirms all previous hypotheses. In particular, the polyester bonds do not play visible role during the compression test and the fibres do not deform, whereas the external porosity remains the most important parameter.

This work shows great potential of X-ray microtomography to follow the internal evolution of structure in very fragile material as low-density fibreboards. The analysis was simplified in $1 \mathrm{D}$ and we can expect that further investigations could involve the $3 \mathrm{D}$ behaviour by using $3 \mathrm{D}$ image correlation. For this purpose, we could make use of the richness of the initial grey levels if we take care to preserve the complete information.

\section{Acknowledgements}

We thank the ID19 beamline team (ESRF) and especially X. Thibault. Our thanks also to J. Malvestio for the contribution to the experiment and to J. Belward for the English corrections.

\section{References}

[1] Badel E et al. Quantitative microtomography: measurement of density distribution in glass wool and local evolution during a onedimensional compressive load. Meas Sci Technol 2003;14:410-20.

[2] Bergonnier $\mathrm{S}$ et al. Strain heterogeneities and local anisotropy in crimped glass wool. J Mater Sci 2005;40:5949-54.

[3] Shi S, Gardner DJ. Hygroscopic thickness swelling rate of compression molded wood fiberboard and wood fiber/polymer composites. Compos Part A: Appl Sci Manuf 2006;37:1276-85.

[4] Faessel $\mathrm{M}$ et al. 3D modelling of random cellulosic fibrous networks based on X-ray tomography and image analysis. Compos Sci Technol 2005;65:1931-40.

[5] Lux J et al. Macroscopic thermal properties of real fibrous materials: volume averaging method and 3D image analysis. Int Heat Mass Transfer 2006;49:1958-73.

[6] Kawasaki T, Zhang M, Kawai S. Manufacture and properties of ultra-low-density fiberboard. J Wood Sci 1998;44(5):354-60.

[7] Burgert I, Gierlinger N, Tanja Z. Properties of chemically and mechanically isolated fibers of spruce. Part 1: Structural and chemical characterisation. Holzforschung 2005;59:240-6.

[8] Muller M, Riekel CB. Combined X-ray microbeam small-angle scattering and fibre diffraction experiments on single native cellulose fibres. J Appl Crystall 2000;33:817-9.

[9] Yamamoto H, Kojima Y. Properties of cell wall constituents in relation to longitudinal elasticity of wood. Part 1. Formulation of the longitudinal elasticity of an isolated wood fiber. Wood Sci Technol 2002;36:55-74.

[10] Sahlberg U, Salmen L, Oscarsson A. The fibrillar orientation in the S2 layer of wood fibres as determined by X-ray diffraction analysis. Wood Sci Technol 1997;31:77-86. 
[11] Persson K. Micromechanical modelling of wood and fiber properties. Lund (Sweden): Lund University; 2000. 213p.

[12] Buffière JY et al. Characterization of internal damage in a MMCp using X-ray synchrotron phase contrast microtomography. Acta Mater 1999;47(5):1613-25.

[13] Rasband SW. ImageJ. Bethesda, Maryland, USA: US National Institutes of Health; 1997-2006.

[14] Serra J. Image analysis and Mathematical Morphology. New-York: Academic Press; 1982.

[15] Matheron G. Eléments pour une théorie des milieux poreux. Paris: Masson; 1967.

[16] Sutton MA et al. Determination of displacements using an improved digital correlation method. Image Vision Comput 1983;1(3):133-9.

[17] Hild $\mathrm{F}$ et al. Multiscale displacement field measurements of compressed mineral-wool samples by digital image correlation. Appl Opt 2002;41:6815-28.
[18] Shipsha A, Berglund LA. Shear coupling effects on stress and strain distributions in wood subjected to transverse compression. Compos Sci Technol 2007;67(7-8):1362-9.

[19] Kajberg J, Lindkvist G. Characterisation of materials subjected to large strains by inverse modelling based on in-plane displacement fields. Int J Solid Struct 2004;41(13):3439-59.

[20] Chen DJ et al. Digital speckle-displacement measurement using a complex spectrum method. Appl Opt 1993;32(11):1839-949.

[21] Sawada T, Sakamoto M. High-resolution large-strain measurement of plastically deformed specimen by Fourier phase correlation. Int $\mathbf{J}$ Mech Sci 2007;49(7):861-71.

[22] Oudjehane A, Lam F. On the density profile within random and oriented wood-based composite panels: horizontal distribution. Compos Part B: Eng 1998;29:687-94. 